\section{PS6:121 JUVENILE AND ADULT-ONSET SYSTEMIC LUPUS ERYTHEMATOSUS: A COMPARATIVE STUDY IN A COHORT OF TUNISIAN PATIENTS}

T Ben Salem, I Naceur, M Tougorti, M Lamloum, I Ben Ghorbel, MH Houman. Department of internal medicine, La Rabta University Hospital, Tunis, Tunisia

\subsection{6/lupus-2018-abstract. 164}

Background Systemic lupus erythematosus (SLE) occurs in $10 \%$ to $20 \%$ of cases before the age of 18 . The aim of this study was to describe clinical, biological and immunological features of juvenile-onset SLE and to compare them to adultonset SLE.

Patients and methods It is a retrospective study including 246 patients with SLE (ACR criteria). Patients were divided in two groups: juvenile-onset SLE (onset age $<18$ years) and adultonset (age was between 18 and 50 years). Data were analysed and compared. $\mathrm{P}$ value was considered significant if $<0.05$.

Results Juvenile-onset SLE (jSLE) was diagnosed in 23 women and two men. Adult-onset disease (aSLE) was seen in 167 women and 12 men (no difference in sex-ratio).

Mean age at disease onset was $14.35 \pm 2.85$ years in jSLE group and mean age at diagnosis was $16.25 \pm 4.38$ years. Mean delay from SLE onset to diagnosis was 18 months in jSLE (similar to aSLE).

At disease onset, malar rash $(88 \%$ vs $65.7 \% ; \mathrm{p}=0.025)$ and Raynaud's phenomena $(57.1 \%$ vs $34.3 \%$; $\mathrm{p}=0.044)$ were significantly more frequent in young patients whereas lupus nephritis (36\% vs 36.4\%) and neurological involvements $(12.5 \%$ vs $12.9 \%)$ were as frequent as in jSLE than in aSLE.

During follow up of patients with jSLE, frequencies of clinical manifestations were as follow: cutaneous manifestations (92\%), lupus nephritis (56\%), pericarditis (39\%) and neurological involvements (25\%) and had no statistical differences from aSLE.

Anaemia was noted in $82.6 \%$, leucopenia in $52.2 \%$ of jSLE patients without statistical differences whereas thrombocytopenia was significantly more frequent $(39.1 \%$ vs $20.6 \%$; $\mathrm{p}=0.046)$.

Antinuclear antibodies, anti-DNA and anti-ENA were positive in $100 \%, 81 \%$ and $86.7 \%$ of jSLE respectively (no differences with aSLE). Anti-Sm antibodies were significantly more frequent in jSLE $(92.3 \%$ vs $62 \% ; \mathrm{p}=0.033)$. Infections were diagnosed in $25 \%$ of jSLE (vs 25.9\%).

Corticosteroids and immunosuppressive therapy prescription was comparable in both groups. Mortality rate was higher in jSLE $(23.8 \%$ vs 9.4\%.p=0.065).

Conclusion Mortality rate was higher in young SLE patients although severe manifestations and infections weren't more frequent in this group

\section{PS6:122 LATE-ONSET SYSTEMIC LUPUS ERYTHEMATOSUS: CHARACTERISTICS AND COMPARISON WITH ADULT- ONSET DISEASE}

T Ben Salem, I Naceur, M Tougorti, M Lamloum, I Ben Ghorbel, MH Houman. Department of internal medicine, La Rabta University Hospital, Tunis, Tunisia

\subsection{6/lupus-2018-abstract.165}

Background Systemic lupus erythematosus (SLE) is uncommon after the age of 50 . The aim of this study was to determine clinical, biological and immunological characteristics of lateonset SLE.
Patients and methods We retrospectively analysed 246 files of SLE patients (ACR criteria). Two groups were defined according to age: late-onset SLE (age over 50 years) and adult-onset (between 18 and 50 years). Characteristics of SLE were compared in the two groups. $\mathrm{P}$ value was considered significant if $<0.05$.

Results Thirty four patients with late-onset SLE were studied; 29 women and five men. Adult-onset group included 173 women and 12 men (no difference in sex-ratio between the 2 groups). In late-onset SLE, mean age at disease onset was $56.9 \pm 6.4$ years) and mean age at SLE diagnosis was $58 \pm 6.7$ years. Mean delay from SLE onset to diagnosis was 18.64 months in late-onset group (similar to adult-onset SLE).

At time of SLE diagnosis, malar rash $(25.8 \%$ vs $65.6 \%$; $\mathrm{p}<0.0001)$ and Raynaud's phenomenon (13\% vs $35.5 \%$; $\mathrm{p}=0.033$ ) were significantly less frequent in late-onset group. Renal failure was more frequent in old patients $33.3 \%$ vs $10.5 \%, p=0.005)$ without difference in lupus nephritis frequencies.

During follow up, cutaneous manifestations (36.4 vs $82 \%$; $\mathrm{p}<0.0001$ ), lupus nephritis ( $25 \%$ vs $45.6 \% ; \mathrm{p}=0.03$ ) were significantly less frequent in late-onset SLE.

Arthralgia was seen in $84.8 \%$ of patients (less frequent than adult-onset group without significant difference). Central neurologic involvements (18.2\%), pericarditis (48.4\%) and pleural effusion $(33.3 \%)$ were more frequent in late-onset SLE without significant differences.

In late-onset SLE, anaemia was found in $67.5 \%$, leucopenia in $40.6 \%$ and thrombocytopenia in $26.5 \%$ of patients (no differencies with adult-onset group).

Antinuclear antibodies were positive in all patients with late-onset disease and anti-DNA antibodies were positive in $69 \%$ of patients (similar to other group).

Anti-ENA antibodies were significantly less frequent in lateonset SLE (66.7\% vs $87.5 \%, \mathrm{p}=0.017)$.

Mortality rates was higher in older patients without statistical difference $(20.9 \%$ vs $9 \%$; $\mathrm{p}=0.14)$.

Conclusion Late onset SLE patients had less cutaneous manifestations and lupus nephritis but had higher rates of renal insufficiency and mortality; these can be related to comorbidities.

\section{PS6:123 EXTENDED T2-TIMES IN CARDIOVASCULAR MAGNETIC RESONANCE (CMR) IN PATIENTS WITH SYSTEMIC LUPUS ERYTHEMATOSUS (SLE) AND PERSISTED DYSPNOEA: IS SLE-ASSOCIATED MYOCARDITIS AN UNDERESTIMATED PROBLEM?}

${ }^{1} \mathrm{P}$ Sewerin, ${ }^{2} \mathrm{~V}$ Lachmann, ${ }^{2} \mathrm{M}$ Gastl, ${ }^{2} \mathrm{P}$ Behm, ${ }^{1} \mathrm{R}$ Fischer-Betz, ${ }^{1} \mathrm{~B}$ Ostendorf, ${ }^{1} \mathrm{G}$ Chehab, ${ }^{1} \mathrm{M}$ Schneider, ${ }^{2} \mathrm{~F}$ Bönner. ${ }^{1}$ Heinrich-Heine-University, Dep. of Rheumatology and Hiller Research Unit, University Hospital, Düsseldorf, GERMANY; ${ }^{2}$ Heinrich-Heine-University, Dept. of Cardiology, Pulmology and Vascular Medicine, Düsseldorf, Germany

\subsection{6/lupus-2018-abstract. 166}

Background To investigate the value of cardiovascular magnetic resonance (CMR) and T2-mapping in patients with systemic lupus erythematosus (SLE) and persistent dyspnoea without sings for pulmonary involvement (conventional X-rays and pulmonary function testing) as a possible sign for myocardial involvement.

Methods 11 women fulfilling the ACR criteria for SLE (mean age $37 \pm 14.81$ years, mean disease duration $13.75 \pm 8.47$ years, mean SLEDAI 6.82 \pm ) with persistent dyspnoea (at least 